\title{
Cross-correlation based movement correction method for biomedical dynamic infrared imaging
}

by M. Strakowska, R. Strakowski, B. Wiecek and M. Strzelecki

Institute of Electronics, Technical University of Lodz, Wolczanska 211/215, 90-924 Lodz, Poland, strakowska.m@gmail.com

\begin{abstract}
During dynamic infrared imaging there is a need of temperature measurement along time at a point or area. The paper shows the recent studies using dynamic infrared imaging in medicine and problems of proper data extraction which is the result of an object movement. The method of movement correction based on cross correlation calculated for dynamic thermal imaging is presented. The influence of movement correction on extracted data and point tracking precision is shown. The proposed method has been successfully applied for cold stress diagnosis during breast cancer screening.
\end{abstract}

\section{Introduction}

There are many approaches to use dynamic thermovision imaging in medical application [1,2,3]. The most important and difficult task for sequence of images analysis is an extraction of appropriate data. In most cases there is a need to get measured values from the same point or area of human body surface for each frame. It is quite hard for people to keep a stable position and do not move at all during the measurement. Although, in some cases the position of a part of human body is stabilized, in other cases it is impossible because of breathing, heart beating or other random movement. There are many examples of movement compensation methods in literature in order to extract correct data from the sequence of images, but most of them are implemented in vision systems [4,5] or traditional medical application like mammography, tomography, MRI etc. $[6,7,8,9]$. Only few examples of such methods can be found according to the thermal images. This is still new but very important and necessary topic to deal with. Mainly there are two approaches of movement correction applied in thermovision - combining vision and thermovision system and methods based on markers. Both of them can reach good result in alignment images but have also drawbacks. The first one assume that image processing, segmentation and detecting landmark or markers on the human skin taking place in visible spectrum recording by CCD camera, and then the movement is calculating and appropriate position is applied in thermovision sequence of images $[3,10]$. Very often the landmark is absent and it is hard to estimate the movement even in visible spectrum. If so there should be put special markers on the skin i.e. in melanomas investigation it can be a ring surrounded the suspicious place [3]. Precision in finding Patient who is under examination must be precisely prepared. Movement correction based on markers demand to put special markers on the skin. There should be compromise in set and number of markers with simplicity and precision of movement compensation [11]. The number and position of the markers should be described and be the same for all patients. Special software with many sophisticated image processing methods should be implemented and very often it can take a lot of time to get appropriate data.

Proposed method of movement correction for thermographic images does not need any special preparation before starting the measurement and work fast. In medical infrared screening it works very well and it is universal algorithm for different type of investigations. Temperature can be measured from every part of human body we want to. To improve movement compensation the small pieces of aluminum sticker can be used but it is not necessary and it is not important where this stickers should be put on the skin.

\section{Proposed method}

Proposed movement correction algorithm is based on normalized cross - correlation [12]. The approach, also called pixel base method, is a similarity measure or match metric. It is well known and widely used visual image processing method [4,5]. Some efforts were already made to use it in infrared imaging [13]. The method compares two images, the template and the base image. The template is a reference image, which position, or an area most similar to it, is localised in base image. The normalized cross - correlation function computes the output matrix of correlation coefficients, which size is the same as the size of the base image and takes values from the range $\langle-1,1\rangle$. The highest absolute value of the coefficient defines the position of the area in image which is the most similar to the template. The implementation follows the formula:

$$
\gamma(u, v)=\frac{\sum_{x, y}\left[f(x, y)-\bar{f}_{u, v}\right][t(x-u, y-v)-\bar{t}]}{\left\{\sum_{x, y}\left[f(x, y)-\bar{f}_{u, v}\right]^{2}[t(x-u . y-v)-\bar{t}]^{2}\right\}^{0.5}}
$$


where:

$f$ is an image,

$t$ is a template image,

$\bar{t}$ is the mean of template,

$\bar{f}_{u, v}$ is the mean of $f(x, y)$ in the region under template.

The normalized cross-correlation method is sensitive for the intensity gradient in the image. It finds successfully the location of the template when it contains characteristic pattern, such as edge, peak of intensity, etc. Because of the fact that all of the matrixes (template, base, output) are rectangular windows there are some limitations. Only horizontal and vertical translation of the template in the image can be efficiently estimated. More complex transformations of image (i.e. rotation) can prevent appropriate localisation the region of interest [4].

In dynamic infrared imaging the thermal response of the subject under test is measured. Appropriate measurement of temperature value change along time in an area or point is vital. In biomedical application this task is difficult as man is unable to hold still along whole procedure, sometimes for several minutes. Moreover, some movements cannot be controlled by patient. Due to this fact, there is need of movement correction in dynamic thermovison, biomedical examinations.

The proposed method calculates the position of measurement point by the average translation of reference points. It assumes that the camera's Field Of View (FOV) observe not only the study area on the body, but also surrounding skin which was not bring under thermal excitation. The surroundings, more or less thermally stable along procedure, is used to determine the body movement.

The flowchart of developed algorithm is presented figure 1. It can be divided into two blocks: the definition of input data and actual movement correction.

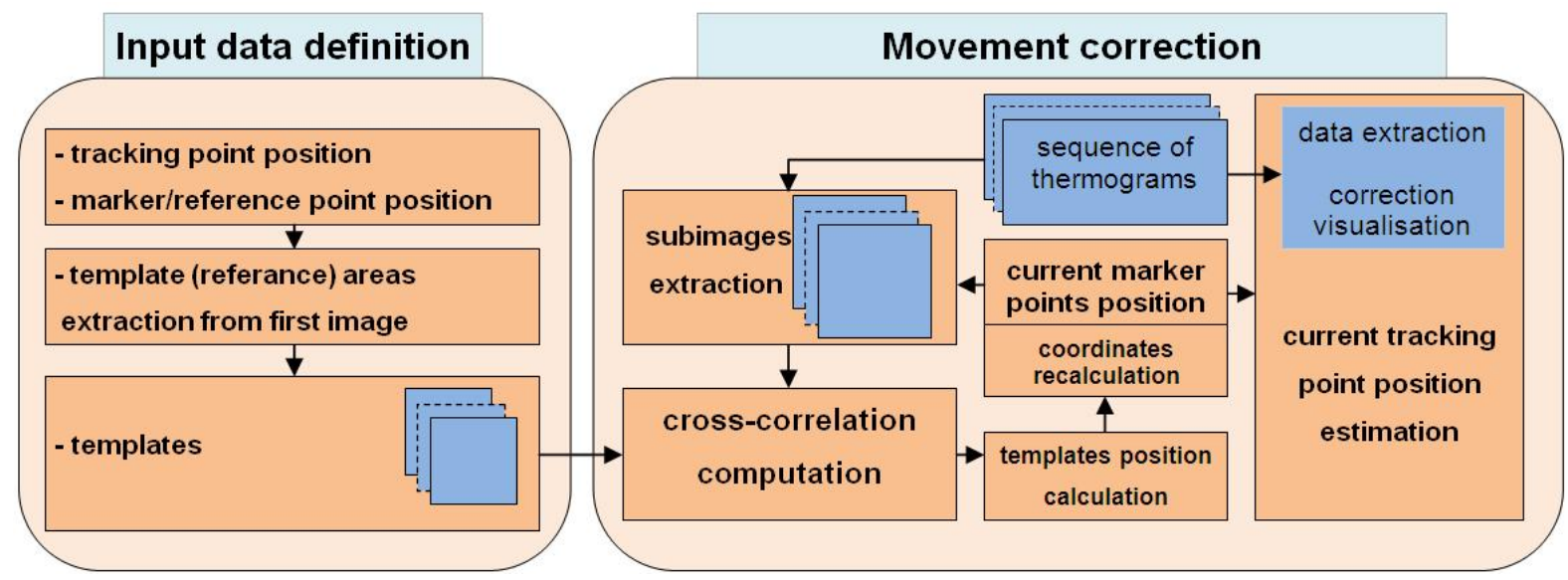

Fig. 1. Developed algorithm flowchart

Starting the work with program proper data must be defined manually: tracking and reference points position. The tracking point is the measurement location where the temperature change is observed. The position of marker points should be located outside the area of skin under thermal excitation to maintain stable temperature. They define the center of templates areas, which are used to determine image translation. After points selections automatic extraction of templates, reference areas, from the first frame of sequence is done. Appropriate selection of marker points is crucial for high algorithm effectiveness. The main assumptions while selecting these points are as follows:

- point is not inside thermally excitated area,

- $\quad$ no significant change of temperature around selected point,

- $\quad$ the best points are selected with gradients of temperature around it, i.e. edges of the body, hot spots on skin caused by blood vessels, etc.

After data input preparation the movement correction is carried out on recoded infrared image sequence. The new tracking point position is calculated as the average translation of all templates areas in both vertical and horizontal direction. In order to determine the position of templates in next image from sequence the normalized cross-correlation (CC) coefficient is computed for all of them. The movement of human body in the FOV is not fast. This causes the maximum translation of few pixels per frame. Taking this fact under consideration to improve the efficiency of algorithm only subimages of infrared frame are taken to calculate CC. Center position of each base subimage is defined as the marker point position from last calculated frame. After calculating CC matrix and finding coordinates of its maximum absolute value, the new position of each marker is known. Based on them current tracking point position is estimated. The size of template must be smaller than the base image for CC calculation. During work on algorithm thermograms from two IR cameras were taken under consideration. There definitions were $288 \times 384$ and $480 \times 640$ pixels. The size of 
template/base subimage was set respectively 25x25/50x50 pixels for lower definition system and 30x30/60x60 for higher definition system. The example of algorithm's works as time-lapse images from sequence is presented in figure 2.

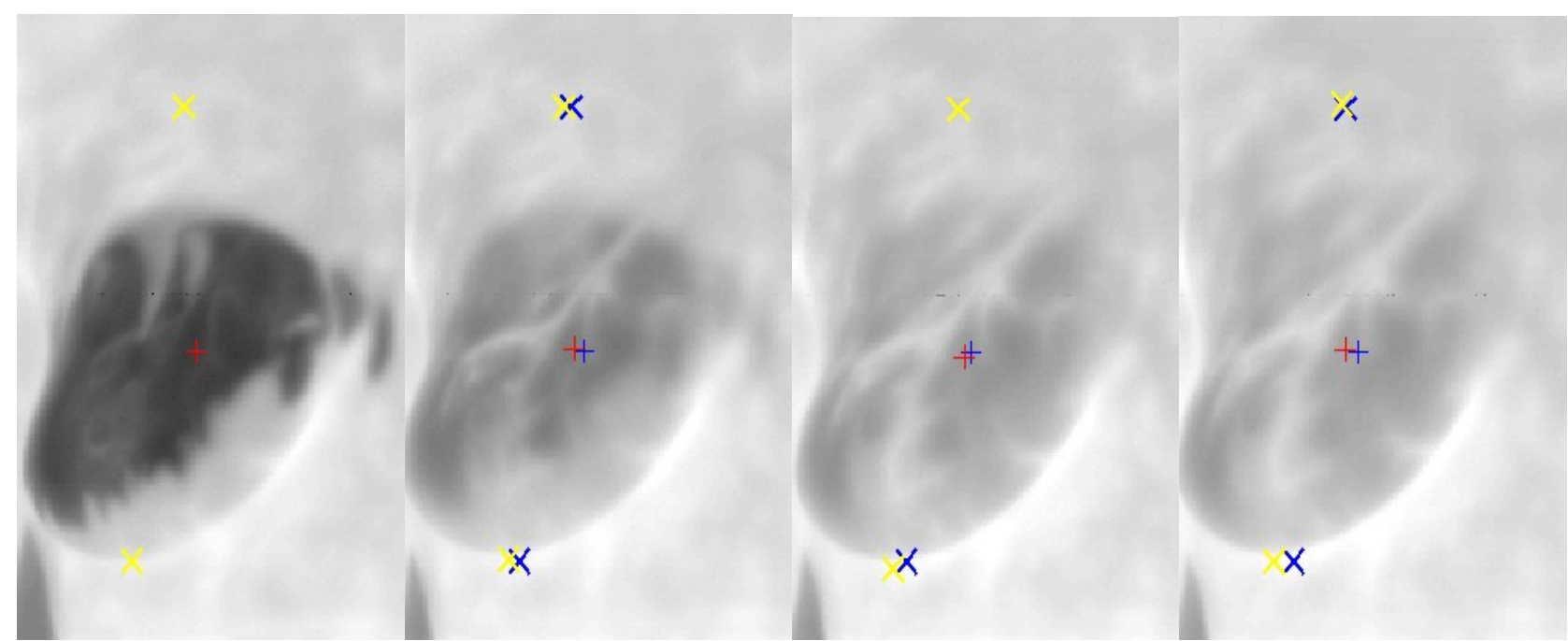

Fig. 2. Example of developed algorithm work with marked points

(blue plus - measurement point start position, red $X$ - measurement point corrected position, blue $X$ - marker point start position, yellow $X$ - marker point corrected position)

\section{Results and discussion}

In dynamic infrared imaging the thermal response of studied object is usually described by exponential function. The same idea is applied to biomedical thermovision to describe thermal response of skin [3]. In such measurements the most important parameter is time constant $\mathrm{T}$, which is obtained from approximation of measured data with exponential function. Therefore, the precision of data acquisition is crucial for proper time constant estimation.

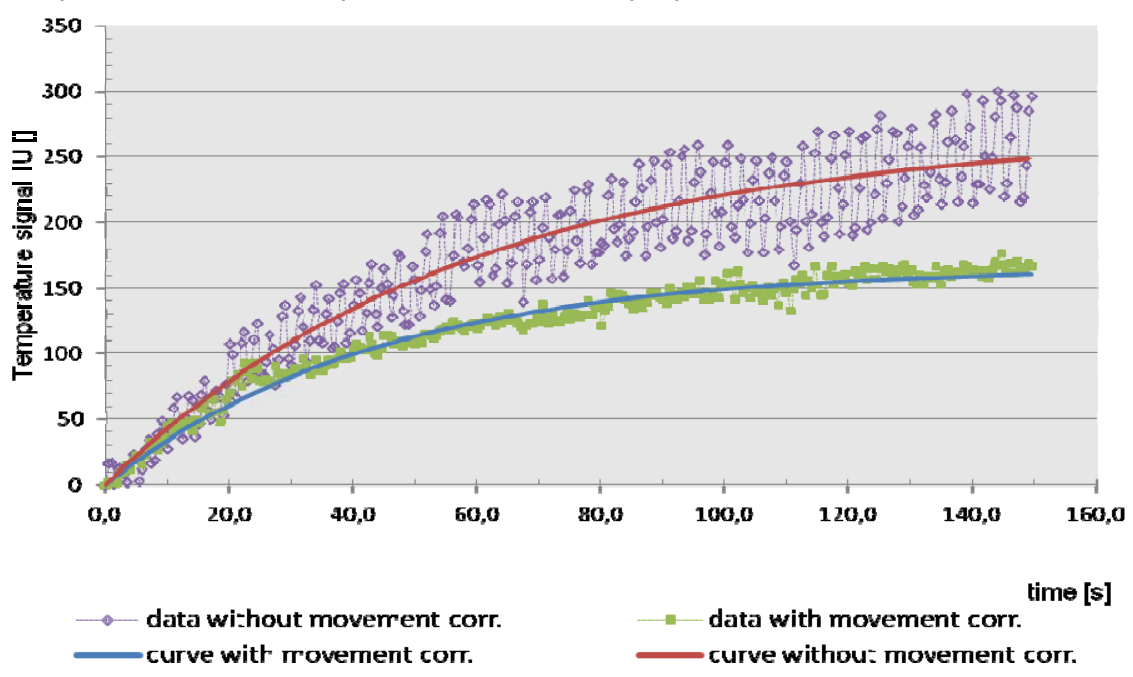

Fig. 3. Measured data values with and without use of movement correction method and its approximated exponential curves

Designed movement correction method has been tested using the sequences of thermograms made during the cold stress for breast cancer screening. The thermal excitation is described as unit step function. After removing thermal excitation the temperature rise caused by body thermoregulation and convection is measured. The main problem in this case was the movement caused by breathing. The 7 sets of data was collected from 4 patients. The measurement points were selected randomly inside an area under thermal excitation to study movement correction method. It is reported that the size of temperature measurement area cannot be only single point (pixel) in medical applications [14]. Therefore, average thermal signal from an area of $5 \times 5$ pixels around selected start points were extracted with and without movement correction. Every dataset describing thermal excitation were fitted to mathematical model of exponential curve and deviation of data samples was calculated. The plot of temperature dependence of time for one point with and without movement correction along with approximated curve is presented in figure 3. Temperature is expressed in isothermal 
units acquired from infrared camera and relative increase. and standard deviation of data samples was calculated. Figure 4 shows the plot of residuals for this point.

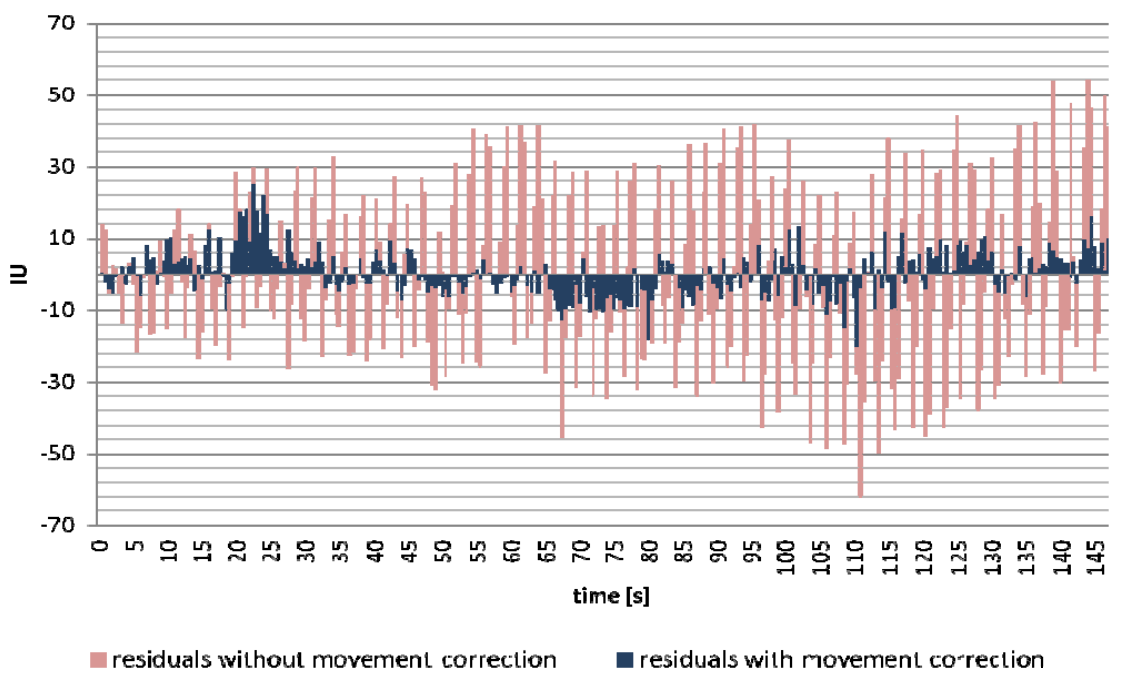

Fig. 4. Residuals plot of data acquired with and without movement correction

The standard deviation and correlation coefficient for exponential curves and data were calculated and presented in table 1. Data acquired with developed movement correction method characterised rise of temperature described by mathematical model more accurately. The average value of standard deviation is two times smaller with movement correction. Moreover, correlation values for every measurement are higher with use of developed method. The character of curves described by the determined value and time constant also change significantly. Due to this fact, it is very easy to make a mistake in measurements without movement correction. Applying the movement correction caused in most cases decrease value of time constant.

Table 1. Parameters of measured data compared with its exponential approximation

\begin{tabular}{|c|c|c|c|c|c|c|c|}
\hline Measured point no. & 1 & 2 & 3 & 4 & 5 & 6 & 7 \\
\hline \multicolumn{8}{|c|}{ Standard deviation, IU } \\
\hline without movement correction & 35,99 & 24,46 & 39,95 & 16,36 & 41,48 & 33,59 & 24,88 \\
\hline with movement correction & 14,00 & 6,52 & 13,88 & 10,79 & 35,46 & 9,87 & 20,61 \\
\hline \multicolumn{8}{|c|}{ Correlation } \\
\hline without movement correction & 0,94 & 0,94 & 0,93 & 0,99 & 0,97 & 0,94 & 0,99 \\
\hline with movement correction & 0,98 & 0,99 & 0,98 & 0,99 & 0,98 & 0,97 & 1,00 \\
\hline \multicolumn{8}{|c|}{ Determined value, IU } \\
\hline without movement correction & 418 & 267 & 404 & 403 & 735 & 463 & 842 \\
\hline with movement correction & 255 & 166 & 294 & 365 & 727 & 171 & 829 \\
\hline \multicolumn{8}{|c|}{ Time constant, s } \\
\hline without movement correction & 39,32 & 57,40 & 43,68 & 35,20 & 22,45 & 110,82 & 32,33 \\
\hline with movement correction & 25,15 & 43,76 & 25,15 & 28,60 & 26,89 & 26,64 & 25,37 \\
\hline
\end{tabular}

Additional tests with marker on the body surface were made to calculate and present the error of movement correction method and the same body point localisation. The test was carried out for two markers positions and different numbers of reference points (templates). The body markers were made by applying the pieces of thin, aluminium foil to the skin. As the aluminium has different emissivity coefficient is clearly visible in infrared image. The correct positions of body markers were estimated in semi-manual method by defining the geometrical center of body markers area. 
Secondly, position of markers was tracked by developed movement correction method. The start position of tracking point was defined as geometrical center of body marker in the first frame of sequence. The tests were carried out with 4 , 3 and 2 numbers of reference points (templates). The effectiveness of body marker localisation is presented in table 2. The best results of movement correction were achieved for 3 templates areas formed in triangle shape around the tracking point. In this case the Euclidean distance error was estimated on average level of 1.68 pixels.

Table 2. Euclidean distance error in pixels for localisation test measurements

\begin{tabular}{|c|c|c|c|c|c|c|}
\hline Body marker no. & \multicolumn{3}{|c|}{$\mathbf{1}$} & \multicolumn{3}{c|}{$\mathbf{2}$} \\
\hline Number of templates & 4 & 3 & 2 & 4 & 3 & 2 \\
\hline Mean error & 2,07 & 1,88 & 2,16 & 2,12 & 1,68 & 1,73 \\
\hline Standard deviation & 0,99 & 0,73 & 0,97 & 0,84 & 0,79 & 0,83 \\
\hline Median & 2,24 & 2,00 & 2,24 & 2,24 & 1,41 & 1,41 \\
\hline
\end{tabular}

\section{Conclusion}

This paper shows the influence of developed movement correction method in dynamic biomedical infrared imaging technique. Our proposal is based on cross-correlation approach. It works well, although the special attention has to be paid for choosing the right reference template. Is certain cases when the image does not contain the characteristic patterns, or it is noisy, the additions markers (typically cold) have to be placed on the patient's skin. This work is applied in the screening protocols for skin and breast cancer treatment.

\section{REFERENCES}

[1] W. C. Amalu, "Nondestructive Testing of the Human Breast: The Validity of Dynamic Stress Testing in Medical Infrared Breast Imaging", Proceedings of the 26th Annual International Conference of the IEEE EMBS, San Francisco, September 2004.

[2] M. J. Janicek, G. Demetri, M. R. Janicek, K. Shaffer, M. A. Fauci, "Dynamic infrared imaging of newly diagnosed malignant lymphoma compared with gallium-67 and fluorine-18 fluorodeoxyglucose (FDG) positron emission tomography", Technology in Cancer Research \& Treatment, vol. 2, no. 6, p. 571-577, December 2003.

[3] T. M. Buzug, S. Schumann, L. Pfaffmann, U. Reinhold U, J. Ruhlmann, "Functional Infrared Imaging for SkinCancer Screening", Proceedings of the 28th IEEE EMBS Annual International Conference, New York, 2006

[4] B. Zitová, J. Flusser, "Image registration methods: a survey", Image and Vision Computing 21, p. 977-1000, June 2003.

[5] M. P. Deshmukh, U. Bhosle, "A survey of image registration", International Journal of Image Processing, Vol. 5, Issue $3,2011$.

[6] M. Zaitsev, C. Dold, G. Sakas, J. Hennig, O. Speck, "Magnetic resonance imaging of freely moving objects: Prospective real-time motion correction using an external optical motion tracking system", Neurolmage 31, p. 1038 1050, 2006.

[7] T. Kober, R. Gruetter, G. Krueger, "Prospective and retrospective motion correction in diffusion magnetic resonance imaging of the human brain", Neurolmage 59, p. 389-398, 2012.

[8] E. H. W. Meijering, W. J. Niessen, J. Bakker, A. J. van der Molen, G. A. P. de Kort, R. T. H. Lo, W. P. T. M. Mali, M. A. Viergever, "Reduction of Patient Motion Artifacts in Digital Subtraction Angiography: Evaluation of a Fast and Fully Automatic Technique", Radiology, Vol. 219, No. 1, p. 288-293, April 2001.

[9] R. R. Fulton, S. R. Meikle, S. Eberl, J. Pfeiffer, Ch. J. Constable, M. J. Fulham, "Correction for Head Movements in Positron Emission Tomography Using an Optical Motion-Tracking System", IEEE Transactions on Nuclear Science, Vol. 49, No. 1, February 2002.

[10] V. Agostini, A. Ala, R. Bussone, A. Ciarlini, S. Delsanto, F. Molinari , E. Possolo, C. Zanon, "Dynamic infrared imaging for breast cancer diagnosis: a feature based registration approach", IEEE International Workshop on Medical Measurement and applications, Benevento, Italy, April 2006.

[11] V. Agostini, M. Knaflitz, F. Molinari, "Evaluation of different marker sets for motion artifact reduction in breast dynamic infrared imaging", Proceedings of the 29th Annual International Conference of the IEEE EMBS, Cité Internationale, Lyon, France, August 23-26, 2007.

[12] J. P. Lewis, "Fast Template Matching". Vision Interface, Vol. 95, p. 120-123, Canadian Image Processing and Pattern Recognition Society, Quebec City, 1995.

[13] A. Bal, M. S. Alam, "Automatic Target Tracking in FLIR Image Sequences Using Intensity Variation Function and Template Modeling", IEEE Transactions On Instrumentations And Measurements, vol. 54, no. 5, October 2005

[14] E. F. J. Ring, H. Mcevoy, A. Jung, J. Zuber, G. Machin, "New standards for devices used for the measurement of human body temperature", Journal of Medical Engineering and Technology, vol. 34, p. 249-253, May 2010 